\title{
Analysis on the Effect of the Cognitive therapy on the Recovery of the Affective Disorder
}

\author{
Jinling $\mathrm{Qi}^{1, \mathrm{a}}$, Lihong Zhang ${ }^{1, *}$ \\ ${ }^{1}$ Institution of Mental Health, Qiqihar Medical University, Heilongjiang, 161006,China. \\ aqiqi@126.com
}

*Corresponding author zhang6868@126.com

\section{Keywords: Cognitive therapy; Affective disorders; Recovery}

\begin{abstract}
Objective: To observe and evaluate the impact of the cognitive therapy on the recovery of the affective disorder. Methods: We choose the recovery of 96 cases patients with affective disorder from September 2013 to November 2014 selection in our hospital, and they were given the routine drug intervention and the cognitive therapy, intervention cycle for 3 months. Results: After the intervention with the cognitive therapy, the mood, personality, adaptation, interpersonal, cognitive dimension scores of the patients were significantly lower, after the intervention in patients with objective support, subjective support and support utilization degree scores were significantly increased, it was compared the clear difference with before intervention $(\mathrm{P}<0.05)$. Conclusion: The application of cognitive therapy in affective disorders recovery can alleviate mental status, increase social support, improve prognosis, and is worthy of clinical popularization and application.
\end{abstract}

\section{Introduction}

The Affective disorders belong to a kind of mental disease, mostly trauma causes mental illness, is often recurrent, severe can lead to patients of severe psychiatric disorders, poses a great burden for the family and society. In treatment, although drugs can improve many positive symptoms of affective disorders, and effective for relapse prevention, there is still residual symptoms and social functions of damaged. The used family and social support to supplement drug maintenance treatment; the prognosis is particularly important for affective disorders. The family and social support is one of the research and application of the more psychological therapy, studies suggest that family and social support can improve the mental symptoms of schizophrenia patients, cognitive impairment, and social function. For concrete, the paper discusses the family and social support for the recovery, the influence of affective disorder is reported as follows.

\section{Materials and Methods}

\section{Materials}

We choose the recovery of 96 cases patients with affective disorder from September 2013 to November 2014 selection in our hospital, 54 cases are male, 42 cases are female; Youngest 18, 36, largest average $(9.3 \pm 2.3)$; Average duration $(1.4 \pm 0.3)$ years. Inclusion criteria: affective disorders diagnosis standards; Is currently in clinical recovery rate of PANSS score 60 minutes or less; 16 the age of 40, primary school education level above; Patients' informed consent. Exclusion criteria: serious body disease or adverse drug reactions; With mental retardation or organic brain disease.

\section{Methods}

All patients were given routine drug intervention and cognitive therapy, intervention cycle for 3 months. Cognitive behavioral therapy basic technology: (1) cognitive technology: mainly includes helping patients to understand automatic thinking, namely the application of the theory of ABC to the patients with abnormal reaction for inciting events with patients between the role of faith, so as to help patients to realize automatic thinking and influence; List the patient's distorted cognition, so as to improve their cognitive level, correct erroneous ideas; Change in patients with extreme faith; Help patients to test hypothesis, through inspection support and the evidence does not support a 
false assumption, help patients know the truth, find themselves cognitive distortion and negative one-sided attitude towards things; Positive self-talk law requires patients to their own negative thoughts, put forward the idea of positive; Homework method such as the patient record in note form one's own thinking and the analysis of the thinking, so as to achieve correct cognitive way. (2) behavior have daily activity plan, arrange some activities can be done to the patient, every hour of every day in a planned way and task, the difficulty of the activities and requirements with the patient's ability and improve and improve mood; Difficulty and feelings evaluation technology, often combined with daily activity plan, use fill in the form of daily activity records, let patients to patients found problems, analyzing problems and encourage its solve problems; , a sequence of relaxation training method, in conjunction with the music of the guide patients to relax certain parts, in turn to fully experience the feelings of tension and relaxation.

\section{Statistical processing}

With mathematical statistics software SPSS19.0 for data collection and statistical analysis, and chi-square test, $\mathrm{P}<0.05$, significant difference has statistical significance.

\section{Results}

Cognitive therapy after intervention in patients with personality, emotion, adaptation, interpersonal, cognitive dimension scores were significantly lower, after the intervention in patients with objective support, subjective support and support utilization degree scores were significantly increased, the clear difference compared with before intervention $(\mathrm{P}<0.05)$.

Table 1: Before and after the intervention of mental state of the Patients

\begin{tabular}{ccc}
\hline Indicators & Before treatment $(\mathrm{n}=96)$ & After treatment $(\mathrm{n}=96)$ \\
\hline Personality & $19.36 \pm 1.23$ & $9.18 \pm 0.21$ \\
Emotion & $22.43 \pm 2.76$ & $10.32 \pm 1.24$ \\
Adaptation & $18.46 \pm 2.65$ & $9.24 \pm 0.93$ \\
Interpersonal & $15.64 \pm 4.38$ & $9.25 \pm 1.34$ \\
Cognitive & $18.23 \pm 2.34$ & $11.25 \pm 0.84$ \\
\hline
\end{tabular}

Table 2: Before and after treatment in patients with social support condition

\begin{tabular}{ccc}
\hline Indicators & $\begin{array}{c}\text { Before treatment } \\
(\mathrm{n}=96)\end{array}$ & $\begin{array}{c}\text { After } \\
\text { treatment }(\mathrm{n}=96)\end{array}$ \\
\hline Objective support & $8.13 \pm 2.14$ & $9.54 \pm 2.18$ \\
$\begin{array}{c}\text { Subjective support } \\
\text { support utilization } \\
\text { degree }\end{array}$ & $19.57 \pm 5.43$ & $21.45 \pm 4.28$ \\
& $7.42 \pm 1.26$ & $8.45 \pm 1.97$ \\
\hline
\end{tabular}

Table 3: Compared before and after treatment in patients with health education effect

\begin{tabular}{cccccc}
\hline Indicators & $\mathrm{n}$ & $\begin{array}{c}\text { Theory } \\
\text { knowledge }\end{array}$ & Self-knowledge & $\begin{array}{c}\text { Consciously } \\
\text { follow the } \\
\text { medical } \\
\text { behavior }\end{array}$ & $\begin{array}{c}\text { Service } \\
\text { satisfaction }\end{array}$ \\
\hline $\begin{array}{c}\text { Before } \\
\text { treatment } \\
\text { After }\end{array}$ & 96 & 85.2 & 72.5 & 72.3 & 80.6 \\
$\begin{array}{c}\text { treatment } \\
\chi 2\end{array}$ & 96 & 97.3 & 93.1 & 95.8 & 100 \\
$\begin{array}{c}\chi^{2} \\
\text { P Value }\end{array}$ & 6.754 & 9.023 & 10.567 & 12.362 \\
& $<0.05$ & $<0.05$ & $<0.05$ & $<0.05$ \\
\hline
\end{tabular}




\section{Discussion}

\section{The affective disorders}

The affective disorders are progressive temporary mental disorders, frequently-occurring disease in young people, in order to temporary changes in personality, memory disorders and mental behavior as the main clinical manifestations. In this disease, the social psychological factors on the occurrence and development of affective disorders there, family and social support is the main content of the social psychological factors. In the recovery period, a lot of patients to their own reality and the surrounding environment have a correct understanding and judgment ability, mood swings, there are different degrees of psychological negative emotions, main performance for different levels of anxiety, depression, social and family status concerns, etc., serious can appear extreme, adventure and suicidal behavior, is not conducive to recover. Therefore need to be positive family and social support, help patients in a timely manner to eliminate trapped emotions such as anxiety and depression, helps the psychological rehabilitation of patients with affective disorder, to return to the family and society has very important significance. In specific interventions, the first thing to make patients feel the respect and understanding, improved their disease, and to the life, the rational understanding of the emotional, and improved their treatment for mental illness and improve self-confidence, promote contact and interaction between the patients with the surrounding environment. To participate in team activities of patients with affective disorder was gradually established in place for close friends of the relationship between, and in the process of sharing peer response, support and help, has played a good emotional catharsis and guidance role.

It is also called affective disorder and mood disorders, it is a significant and lasting change mood; the mood is high or low as the basic clinical manifestations, accompanied by the corresponding thinking and behavior change, tend to break out repeatedly, break complete remission. Although several episodes, after proper treatment, most mental activity is not a recession, prognosis is good. Affective disorder prevalence was $0.76 \%$, in order to see more women, this may be related to stress more than women, often under experience negative life events, and the lack of effective countermeasures to deal with. The cause of the disease is unknown, most research suggests that the disease and psychosocial factors, genetic factors, biological factors and physical factors. The weight of the affective disorder degree and the recurrence rate is different from person to person, can vary widely, light person can work as usual, not heal, or attack only $1 \sim 2$ times in my life, the person that weigh not only require hospitalization, and even life-threatening, frequent attacks. In affective disorders are mainly divided into clinical mania, bipolar affective disorder, depression and mood disorders and so on.

According to relevant research shows that, at present in the psychiatric community, the diagnosis of affective disorder standard is very low, but the diagnosis of schizophrenia is constantly expanded, then that there's a certain deviation in diagnostic criteria, as well as the related concepts of disease appeared insufficient understanding, etc., makes the misdiagnosis appear constantly. Affective disorder patients were misdiagnosed as schizophrenia is mainly due to dominated by affective disorder symptoms and some disruptive symptoms patients appeared. On the patient during the diagnosis, because the doctors ignored the emotional symptoms of patients, just notice the disruptive symptoms of patients, eventually leading to misdiagnosis. Therefore, the patient during the diagnosis, the spiritual symptom is not enough to just study cross-sectional confirmed, due to one or several psychiatric symptoms can appear in different nature of the disease, therefore, not only to patients if disruptive symptoms to diagnose patients. It also shows that the doctor insufficient understanding of related concepts, this is also the cause of misdiagnosis. In the past, to the concept of diagnosis such as manic depression, just see whether patients with obvious symptoms of "three high three low" to judgment as the basis. When patients with hallucinations and delusions and logical barriers such as the so-called disruptive symptoms, regardless of whether patients with obvious symptoms of three patients diagnosed with schizophrenia. 


\section{The characteristics of the cognitive behavioral therapy}

The concept of cognitive behavioral therapy is based on cognitive theory, the basic reasons for a personal behavior and emotional responses, rather than the event itself one view of events, ideas, interpretation and evaluation, namely belief of such an event, in the case of the objective facts cannot be changed, individuals can completely by changing the cognitive methods to improve their mood and behavior. The therapy is simple, saving time, quick effect, its simplicity type depends on the good operability and homework treatment, namely leave homework after each psychological treatment, encourage cooperation and deepen the treatment, patients used as homework way to strengthen the treatment effect, can greatly improve the treatment effect, generally only average accept 16 patients with treatment can recover gradually. Using heuristic method of education, the key does not lie in correcting the bad behavior itself, but in the correct to oneself, others and the surrounding environment and unreasonable faith of things, and these irrational beliefs have asked absolute, overly generalization, and horrible three common characteristics. So treatment is to change the irrational ideas, through a series of analysis, argument, go directly to change the philosophy of life, to correct the unreasonable way of thinking, instead of the correct way of thinking and life belief system, and formed a new world view. And adopt the method of inquiry, and insight into the thoughts and ideas of patients, according to the different cultural level of patients, properly using popular language and jargon, combined with the corresponding answer, explore the irrational beliefs of patients, the logic of the thoughts and feelings, inappropriate behavior response relationship. In cognitive behavioral therapy, the patient is the role of the executor of the narrator, the learners and the learning process, and the healer is listener, encourage and educators, therefore the healer to establish good doctor-patient relationship with the patient, kind, sincere attitude, caring respect patients, for patients to keep a secret. Because in many cases, the prognosis of patients with depends on how well the doctor-patient relationship, the therapist's task is trying to get patients learn to abandon the idea of before, at the same time to learn a new way to deal with, is good at discover patients life goals and help them achieve the goal, to establish the cognitive behavioral therapy specific mode of education, bad mood and behavior reaction of most of the patients, corrected by learning.

\section{The effect of the ognitive therapy on the recovery of the affective disorder}

The cognitive behavior therapy is a relatively young, with its unique way of treatment, can correct the patient's thoughts, beliefs and attitudes, so as to achieve therapeutic purposes, not only have important theoretical basis, and the implementation of the scientific technology and skills, in the psychiatric clinical application are confirmed, the therapy is a new mode conforms to the biological - psychology - social medicine of a kind of new method. Research proved the feasibility of cognitive behavioral therapy is simple operation, save medical resources, quick effect and durable, easy to promote. Suitable for a variety of mental illness, which is beneficial to the recovery of patients with comprehensive and system? Cognitive behavior therapy for depression research: Favg will randomly 40 cases such as the antidepressant treatment of remarkable progress of heavy depression patients, divided into cognitive behavioral therapy group and routine clinical treatment group, continue to treat residual symptoms and gradually reduce the antidepressant drugs to stop. Two years follow-up study showed that the level of residual symptoms cognitive behavioral therapy group was obviously lower routine clinical treatment group, at the same time reduce the recurrence of depression (cognitive behavioral therapy group recurrence rate was $15 \%$, the routine clinical treatment group is 35\%). The findings continue to 4 years of follow-up, cognitive behavioral therapy group recurrence rate was 35\%, the routine clinical treatment group (70\%)\}. Cognitive behavioral therapy can decrease disability symptoms, reduce the recurrence rate, reduce the recurrence rate.

Cognitive behavior therapy for depression after schizophrenia research: Jiang Kai-da, watch the video "" prevention of depression research, a total of 8 class, every class 2030 min 23 cases of secondary depression on cognitive behavior therapy to treat patients with schizophrenia, results in patients with Hamilton depression scale, called depression self rating scale and brief psychiatric 
scale, gross rating scale score has gradually reduce the trend, the more the more obvious curative effect.

Cognitive therapy: (1) to help build and support to adapt to the sexual function, correct the patient's own system of prejudice. Remove note or distracting, let patients learn to relax, breathing exercises control and adhere to the principle of not to avoid, at the same time try to use positive language suggested to replace the original negative cognition and ideas such as beep (2) to help identify patients with negative ego thoughts, helping patients to realize external events and individual emotional experience, behavior; the relations between (3) to help patients tested negative automatic thoughts, namely the healer and patient with the patients of automatic type thought as a kind of hypothesis test them, by the idea of system and sharp questions make patients to review the correctness of their ideas, change the negative thoughts; (4) help to identify patients with dysfunctional key assumptions, negative automatic thoughts results from the function key assumptions, so once the patients of treatment can more skillfully to identify and test the automatic thoughts, the key point of treatment should be turned to work on the assumption of the function of tonal, and because these assumptions are the factors influencing patients, but it is not detected by consciousness, identifying these assumptions often adopt the method of inference; (5) plate clean function of tonal assumes that the recognition of tonal hypothesis is to make the change, and make its produce change method should be adopted; For patients with cognitive homework produce cognitive change is an effective method.

\section{Conclusion}

The cognitive behavior therapy is a relatively young therapy, with its unique way of the treatment, and it can correct the patient's thoughts, beliefs and attitudes, so as to achieve therapeutic purposes. It not only have important theoretical basis, and the implementation of the scientific technology and skills, in the psychiatric clinical application are confirmed, the therapy is a new mode conforms to the biological - psychology - social medicine of a kind of new method. The application of cognitive therapy in affective disorders recovery can alleviate mental status, increase social support, improve prognosis, and is worthy of clinical popularization and application.

\section{Acknowledgement}

This research was financially supported by the National Science Foundation.

\section{References}

[1] K. Wilson-d'Almeida, A. Karrow,M.-C. Bralet, N. Bazin,M.-C. Hardy-Baylé, B. Falissard. In patients with schizophrenia, symptoms improvement can be uncorrelated with quality of life improvement[J]. European Psychiatry . 2011(5).

[2] Stefan Priebe, Rosemarie McCabe, Ulrich Junghan, Thomas Kallert, Mirella Ruggeri, Mike Slade, Ulrich Reininghaus. Association between symptoms and quality of life in patients with schizophrenia: A pooled analysis of changes over time[J]. Schizophrenia Research . 2011 (1). [3] Alessandro Rossi. Attitudes toward medication and the clinical variables in schizophrenia: Structural equation models[J]. Patient Preference and Adherence . 2012 (1).

[4] Alex M. Wood, Stephen Joseph, Joanna Lloyd, Samuel Atkins. Gratitude influences sleep through the mechanism of pre-sleep cognitions[J]. Journal of Psychosomatic Research . 2012 (1). [5] David P. Folsom,Colin Depp, Barton W. Palmer, Brent T. Mausbach, Shahrokh Golshan, Ian Fellows, Veronica Cardenas, Thomas L. Patterson, Helena C. Kraemer, Dilip V. Jeste. Physical and mental health-related quality of life among older people with schizophrenia[J]. Schizophrenia Research. 2013(1).

[6] Thomas L. Patterson, Oscar R. Leeuwenkamp. Adjunctive psychosocial therapies for the treatment of schizophrenia[J]. Schizophrenia Research . 2007 (1). 
[7] A. Riecher-Rössler, U. Gschwandtner, S.Borgwardt, J. Aston,M.Pflüger, W. Rössler. Early detection and treatment of schizophrenia: how early?[J]. Acta Psychiatrica Scandinavica . 2012(2). [8] Cheng-Fang Yen, Cheng-Chung Chen, Yu Lee,Tze-Chun Tang,Chih-Hung Ko,Ju-Yu Yen. Insight and correlates among outpatients with depressive disorders[J]. Comprehensive Psychiatry . 2012(5).

[9] Michael F. Green, Robert S. Kern, Robert K. Heaton. Longitudinal studies of cognition and functional outcome in schizophrenia: implications for MATRICS[J]. Schizophrenia Research . 2013 (1).

[10] Celso Arango, Julio Bobes. Managing acute exacerbations of schizophrenia: focus on quetiapine[J]. Current Medical Research and Opinion® . 2013 (5).

[11] Christine Barrowclouch, Gillian Haddock, Fiona Lobban, et al.Group cognitive behavioural therapy for schizophrenia. British Journal of Psychiatry . 2012 (3).

[12] Monika S, Jan P, Daniela J, et al. The quality of life of patients suf-fering from schizophrenia-acomparison w ith healthy controls. Biomed Pap Med Fac Univ Palacky Olomouc Czech Repub . 2012 (5). 